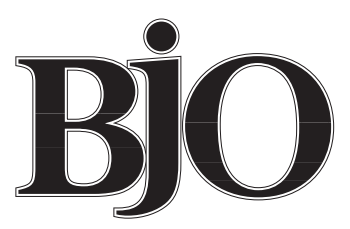

British Journal of Ophthalmology

\title{
Editorials
}

\section{Colour imaging using a scanning laser ophthalmoscope}

New imaging techniques have opened up new possibilities for visualising the living human eye and can provide quantitative measurements of fundus features. One of the most important developments has been based on the scanning laser ophthalmoscope (SLO) which has the property of providing optical sections through the use of confocal optics. ${ }^{12}$ This can reveal three dimensional aspects of structures which would otherwise be invisible owing to the contrast degrading effects of overlying elements. The confocal SLO has had a major impact on research in ophthalmology and continues to break new ground in advancing the limits of seeing and measuring properties of the eye. The first widely used SLO was the Rodenstock device which provided facilities for confocal imaging, fluorescein and indocyanine green angiography, and psychophysical measurements such as microperimetry. These used a variety of lasers of different wavelengths to give real time video images. In comparison with photographic techniques, the spatial resolution of video images is inherently lower. This is because the horizontal scan lines which make up the image are limited to approximately 600 compared with the equivalent of 4000 or more for photographs. The mechanical limitations of the spinning polygon mirror mean that this limit will not be easily improved. Despite this limit the confocal optics of the SLO can result in improved resolution compared with photographs because it allows optical sectioning, and so the contrast degrading effects of structures above or below the depth of interest can be reduced. The depth resolution can be of the order of several hundred micrometres. In this way small vessels or other structural detail obscured by the scattering of neural tissue in the optic nerve can be seen and some of the contrast degrading effects of ocular media opacities can be bypassed. New opportunities continue to appear so that we now are able to see and measure the autofluorescence associated with lipofuscin ${ }^{3}$ and quantify the structure of the lamina cribrosa ${ }^{4}$ using a Zeiss prototype laser scanning ophthalmoscope.

Perhaps the most successful and widely used implementation of the SLO is the Heidelberg retina tomograph (HRT) which is aimed at quantifying the three dimensional shape of the optic nerve head using of a series of optical sections combined with the appropriate computer analysis. ${ }^{5}$ It is evident that this measurement ability is not possible using photographic methods. Although the presentation of the images makes very effective use of colour, this is not intended to be realistic and the use of a single laser makes true colour rendition impossible. The images used are of even lower lateral resolution than conventional video images, having only $256 \times 256$ picture elements (or pixels). This illustrates that, despite the lack of lateral resolution, the depth resolution along with the computer analysis allow the SLO to provide information about fundus structures which would otherwise not be available.

Despite these recent advances, one of the limitations of SLO images has been the lack of realistic colour in the images. Important information such as pallor of the disc or subtle aspects of the colour appearance of drusen, diabetic retinopathy and other fundus features are lost with SLO imaging. Although the depth resolution and the resistance to contrast degrading effects provides an advantage over conventional imaging, the lack of colour has been a severe handicap. This has now been overcome, as shown in the paper by Manivannan and colleagues in this issue ( $p$ 342) which presents the first realistic colour images using the SLO.

In principle, the method is straightforward. The combination of three lasers at appropriate wavelengths to form a colour image is analogous to the formation of colour television images using red, green, and blue phosphors. The use of multiple lasers in an SLO to provide colour information has been recognised before. ${ }^{67}$ However, in practice, achieving realistic colour rendition with an SLO requires careful selection of the appropriate wavelengths for the lasers and overcoming difficult problems in image registration which could distort the image.

This is the first time that such realistic three colour images have been produced and the results are impressive. In comparing the colour SLO images with the photographic images the clarity and colour appearance is striking, particularly considering the difference in lateral resolution. In some cases additional detail may be visible in the SLO images and further experience using depth resolved colour imaging will elucidate the role of this new technique.

Clearly there are some limitations and differences in comparison with colour photography, including the inherent difference in lateral resolution common to all SLOs. In addition, the wavelengths of the lasers used would be expected to have an effect. Each laser emits at a single wavelength while the photographic method in combination with the white light flash gives a broad spectral response. As a consequence some differences could arise in comparing subtle colour rendition. In particular, with yellowing of the lens, the short wavelength blue laser 
could contribute a reduced blue component in comparison with the photographic method. Whether these are significant effects remains to be seen. The advantages of confocal optics of the SLO may outweigh any colour differences.

The use of realistic colour information certainly improves our appreciation of the SLO images. Whether the advantages outweigh the extra cost of an SLO compared with other systems is not known. It may be that the role of the SLO is primarily in quantification of images and in this the use of three simultaneous wavelengths could provide an advantage. For example, the qualitative description of "disc pallor" is amenable to exact measurement with the use of multiple wavelengths and other approaches may prove fruitful. The future role of a full colour SLO remains to be seen but the results presented here are a remarkable visual demonstration of the continuing expansion of new possibilities in imaging.

F W FITZKE

Institute of Ophthalmology, University College London

1 Webb R, Hughes G, Pomerantzeff O. Flying spot TV ophthalmoscope. Appl Opt 1980;19:1991-7.

Webb R, Hughes G, Delori F. Confocal scanning laser ophthalmoscope. Appl Opt 1987;26:1492-9.

3 Ruckmann AV, Fitzke F, Bird A. Distribution of fundus autofluorescence with a scanning laser ophthalmoscope. Br f Ophthalmol 1995;79:407-12.

4 Bhandari A, Fontana L, Fitzke F, et al. Quantitative analysis of the lamina cribrosa in vivo using a scanning laser ophthalmoscope. Curr Eye Res 1997; 16:1-8.

5 Dreher A, Tso P, Weinreb R. Reproducibility of topographic measurements of the normal and glaucomatous optic nerve head with the laser tomographc scanner. Am f Ophthalmol 1991;111:221-9.

6 Webb R. Optics for laser rasters. Appl Optics 1984;23:3680-3.

7 Kobayashi K, Akiyama K. Laser-beam scanning system using an acoustic-optic deflector: its application to fundus imaging. Meas Sci Technol 1990;1:151-7.

\section{Macular holes - a diagnostic and therapeutic enigma?}

In previous times diagnosis of a macular hole appeared to be easily done by biomicroscopy or just by ophthalmoscopy. It was a comparatively rare diagnosis without therapeutic relevance. It is only 10 years ago that specific diagnostic criteria were set to consider surgical intervention. ${ }^{1}$ Further studies have established the usefulness of vitreoretinal surgery for macular holes. Macular hole is now a common diagnosis, affecting almost $3 \%$ of the elderly population. However, diagnostic and therapeutic details remain an enigma.

Today, two types of surgical intervention are used to treat macular holes. One is to perform vitrectomy, remove the vitreous cortex, apply some sort of biological chorioretinal glue ${ }^{2}$ over the macula, and finally to fill the vitreous cavity with a long lasting gas bubble, tamponading the macular hole with the patient in a prone position. The "biological glue" in use may be transforming growth factor $\beta$, autologous serum or plasma, platelet concentrates, thrombin, and others. Another surgical approach is to omit the biological glue and instead remove the internal limiting membrane of the retina including the adhering fibrocellular proliferations. ${ }^{34}$ Both modifications of surgery for macular holes have a very high rate of success in closing the macular hole; the results with regard to functional outcome are not yet comparable in a randomised fashion.

So far, it is not clear whether there are different forms or specific types of macular holes which warrant one or the other type of operation. Also, it is unclear why differing surgical approaches are leading to similar good results. It would therefore be of great interest to have predictive diagnostic criteria to obtain more insight into the pathogenesis of macular hole formation. In short, it is of interest to know whether changes at the vitreoretinal interface or changes at the metabolic level of the retina and/or the retinal pigment epithelium (RPE) are responsible for the formation of a macular hole, thus determining the mode of therapeutic action. Diagnostic tools to answer these questions are most desirable.

It not surprising, therefore, that all available diagnostic tools are used to achieve the most precise diagnostic evalu- ation. So far fluorescein angiography, optical coherence tomography (OCT) and, most recently, the retinal thickness analyser (RTA) have been used. In this issue ( $p$ 346) confocal laser scanning ophthalmoscopy (cLSO) is discussed as a potential alternative in the assessment and differential diagnosis of full thickness macular holes. However, measurement of increased fundus autofluorescence in the area of a macular hole may accurately detect a defect in the shadowing effect of xanthophyll. It may permit an unhindered view of the lipofuscin laden RPE, but this measurement of autofluorescence does not give a clue to any specific pathogenic mechanisms. Thus, cLSO might help to differentiate between true full thickness macular holes and pseudoholes, but does not help to answer the puzzling questions mentioned above. Despite its elegant help in confirming a definite diagnosis, cLSO so far does not have a prognostic value similar to fluorescein angiography.

In order to elucidate major pathogenic factors of macular hole formation and, subsequently, the appropriate treatment comparative evaluation of morphological and functional tests will be necessary. Analysis of any removed tissue $^{5}$ and possibly more insight into the cell biology of glial cell proliferation and posterior vitreous separation are needed to answer the enigma of the macular hole and its therapy.

ANSELM KAMPIK

University Eye Clinic, Munich

1 Johnson RN, Gass JDM. Idiopathic senile macular holes: observations, stages of formation, and implications for surgical intervention. Ophthalmology 1988;95:917-24.

2 Smiddy WE, Glaser BM, Green WR, et al. Transforming growth factor beta: a biologic chorioretinal glue. Arch Ophthalmol 1989;107:577-80.

3 Rice TA. Technique of removal of the inner retinal surface in macular hole surgery (abstract). Annual Meeting of the Retina Society, 1955. Santa Fe, 6-10 September 1955, p 68.

4 Liesenhoff O, Messmer EM, Pulur A, et al. Operative Behandlung durchgreifender Makulaforamina. Der Ophthalmol 1996;93:655-9.

5 Messmer EM, Heidenkummer HP, Kampik A. Ultrastructure of epiretinal membranes associated with macular holes. Graefes Arch Clin Exp Ophthalmol 1998; (in press). 